\title{
The subtle dynamics of liquid sheets
}

\author{
JENS EGGERS
}

Department of Mathematics, University of Bristol, University Walk, Bristol BS8 1TW, UK

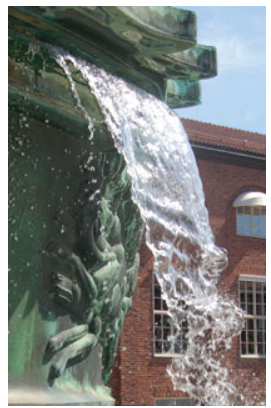

Liquid sheets are a generic occurrence in nature. They provide a key pathway by which liquid matter breaks up and is dispersed into smaller entities, as in the formation of sprays. However, the stability of liquid sheets is surprisingly subtle, and involves a range of physical effects, without which a quantitative understanding is impossible. As a result, even the linear stability of liquid sheets has remained open for over half a decade. Now, by a combination of experiment and thorough theoretical analysis by Tammisola et al. (J. Fluid Mech., this issue, vol. 672, 2011, pp. 5-32), we have finally gained a solid understanding of the key factors that control the initial stages of sheet breakup.

Key words: breakup/coalescence, gas/liquid flow, instability

\section{Introduction}

Linear stability analysis is central to our understanding of how structure arises in hydrodynamics. Perhaps the earliest example is the instability of (round) jets, driven by surface tension, see Eggers \& Villermaux (2008). A reduction in radius lowers the surface energy, and thus drives the jet to breakup and to the formation of form drops: a jet is inherently unstable on account of its geometry. By contrast, sheets (or plane jets) are intrinsically stable; any deformation will lead to an increase in surface area, and is thus energetically unfavourable. Instability must originate either from the rim bordering the sheet or from the interface to another (gas) medium that surrounds the sheet. The focus of the paper by Tammisola et al. (2011) is on the latter case, in which the instability is driven by the difference in speed between the sheet and its surrounding medium, similar to the classical Kelvin-Helmholtz instability (Drazin \& Reid 1981) of a vortex sheet.

Conventional wisdom has it that the onset of instability (described by linear theory) is easier to analyse than the subsequent nonlinear dynamics. One starts from a static configuration such as a sheet, and adds a sinusoidal (either in space or time) perturbation to it. The eigenvalue spectrum of the linearised problem then contains the solution: if all growth rates are negative, the system is stable, otherwise the corresponding (time)-periodic perturbation is amplified. Thus, the hydrodynamic problem at hand, notoriously nonlinear in character, has been reduced to a much simpler linear problem. Paradoxically, however, linear problems can be much harder to interpret than their fully nonlinear counterparts.

The reason is that nonlinear phenomena are universal (they are free of adjustable parameters, Eggers \& Villermaux 2008), while at the linear level the dynamics depend 
crucially on the initial perturbation, such as its spectrum, its initial amplitude, and where it is introduced into the system. Likewise, a nonlinear problem usually involves only a few dominant forces that come in at leading order. On the other hand, the price one has to pay for reducing a nonlinear problem to a linear one is that all effects become coupled, resulting in a complicated system containing many parameters.

From an experimental point of view, the challenges of linear analysis are also considerable. For a linear description to be valid, one must be able to record perturbations of small amplitude and follow them with a considerable dynamic range, in order to pick up the exponential growth determining the growth rates. Thus, however easy it may be to consider a linear stability problem, it may take a very long time to lay it to rest, of which the present problem is a notable example.

\section{Overview}

The origin of the sheet instability, is captured by a calculation neglecting the viscosity of both the sheet and the surrounding gas, but including the Bernoulli suction provided by the gas moving relative to the sheet (Squire 1953). As the sheet deforms in a sinuous manner, an underpressure is created at the crest of the sheet relative to its underside, which is proportional to the deformation amplitude (Villermaux \& Clanet 2002). This destabilising force is opposed by surface tension, which tends to keep the sheet straight. As a result of the interplay between the two forces, instability occurs if the sheet-based Weber number $W e=\rho_{l} U^{2} a / \gamma$ is greater than unity. Here $\rho_{l}$ is the fluid density, $U$ the relative speed between the sheet and the surrounding gas, $2 a$ the sheet thickness and $\gamma$ the surface tension.

Curiously, the same dimensionless combination occurs in the retraction dynamics of a liquid sheet, bordered by a rim (Taylor 1959). While balancing the retraction force $2 \gamma$ with the inertia of the growing rim, one finds a constant speed of retraction $U_{r e t}=\sqrt{\gamma / \rho_{l} a}$. Thus any sheet has $W e>1$, and is unstable when issuing into a stagnant environment, if it is not to be eaten up by its growing rim!

Apart from the sinuous mode in which the sheet deforms at constant thickness, there exists another varicose mode in which the sheet's thickness varies periodically. In the inviscid calculation as well as in most practical situations, however, the sinuous mode dominates. Owing to fluid mass conservation, the varicose deformation requires fluid to be transported over the entire wavelength $\lambda$ of the perturbation, which is much larger than the thickness of the sheet (Villermaux \& Clanet 2002). Indeed, the present experiments clearly show the sinuous nature of the instability (cf. figure 1).

One might think that the most important additional ingredient is the liquid viscosity, since it is larger than the air viscosity. However, the key observation of the present and an earlier companion paper (Söderberg \& Alfredsson 1998) is that the air viscosity changes the base state of the problem in a significant way, as the surrounding airflow forms a boundary layer, whose thickness grows like the square root of the distance $x$ from the nozzle. The inviscid instability comes from the sudden jump of the velocity across the interface, which is now mollified over the width $\delta$ of the boundary layer. Thus, even an almost inviscid environment has a significant stabilising effect on the sheet.

Unfortunately, this implies a significant complication of the theoretical calculation, which has to be done numerically. As the results of Tammisola et al. (2011) reveal, the inviscid theory overpredicts the maximum growth rate of the instability by a factor of 2 , and the growth rate as a function of the excited frequency has a shape quite 
Side views

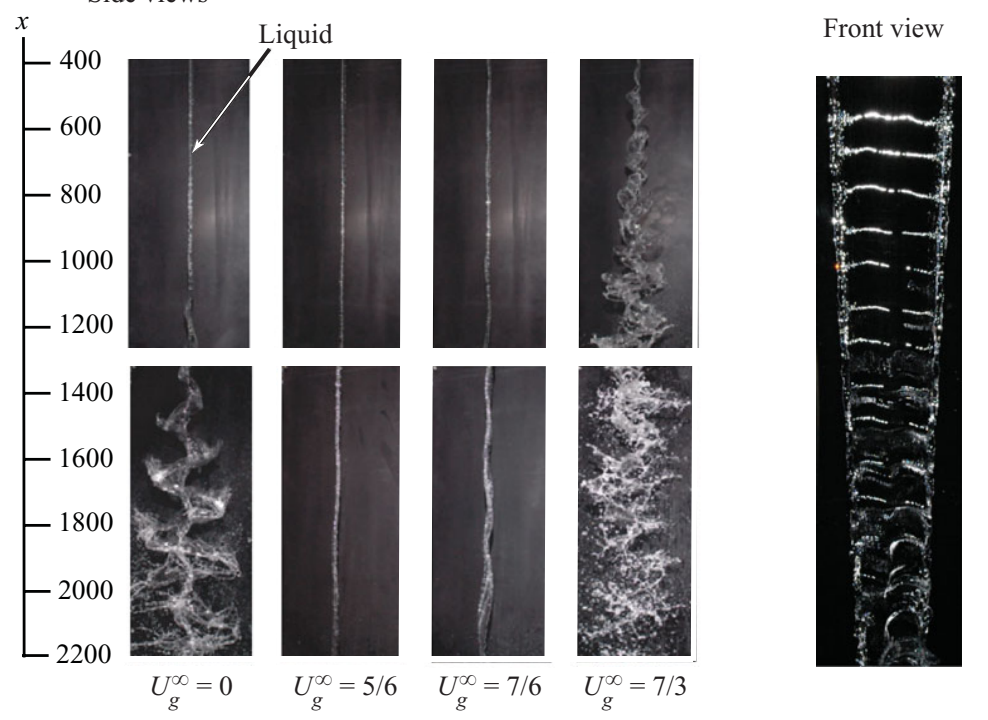

FIGURE 1. Breakup of a sheet of water in a comoving gas stream; $U_{g}^{\infty}$ is the gas velocity normalised by the fluid velocity. A front view shows the breakup into ribbons.

different from the inviscid theory. The numerical results can be neatly summarised by saying that the combined effect of inviscid instability, moderated by a smooth boundary layer, leads to growth rates that are proportional to the velocity difference between sheet and air, and inversely proportional to the square root of the boundary layer thickness $\delta$.

Perhaps the most significant achievement of Tammisola et al. (2011) is the careful experimental measurement of sheet breakup, illustrated in figure 1, and the comparison of the wave growth (without adjustable parameters!) to the numerical results. Upon external forcing the sheet responds by amplifying the corresponding frequency, which is the hallmark of a convective instability. Excellent agreement between numerical and experimental growth rates is found. The leftmost photograph is from a sheet issuing into stagnant air, but the experiments allow for an airstream to co-flow with the liquid. The air speed is increasing from left to right, and the experiments illustrate very nicely that it is the velocity difference which matters. As a result, the instability disappears if the air speed is almost the same as that of the liquid, and grows again if the air speed is significantly faster.

\section{Future}

How does a piece of fluid break up into drops of much smaller size? Few systematic studies of the entire pathway leading to fragmentation exist, but a generic route appears to be via sheets (Villermaux 2007). A simple reason for this is that a sheet is an object of codimension 1, i.e. removed by a single constraint from filling up space. The work by Tammisola et al. (2011) provides a framework on which studies of the subsequent fate of the ubiquitous sheet can be based. Extending our 
previous argument, lines (jets or ligaments in physical terms) are the structures of codimension 2 into which a sheet is expected to evolve. Figure 1 (adapted from Tammisola et al.'s paper) illustrates beautifully how the instability of the sheet leads to an array of ribbons going across the sheet (an alternative route to ligaments is by transformation of the sheet's rim; see Villermaux 2007). The wavelength (spacing between the ligaments) corresponds exactly to the frequency of excitation, proving that linear stability controls the overall structure of the transformation into ligaments.

However, this observation alone does not explain why the dynamics of the sheet proceeds to break up. This gap is filled by an important recent paper (Burton \& Taborek 2007), which shows that the interplay between inertia and surface tension alone can provide the necessary focusing for the sheet thickness to go to zero in finite time, if only a sufficient initial perturbation is present. Near the pinch point where the sheet thickness goes to zero, the sheet profile has a similarity solution,

$$
a(x, t)=\left(t_{0}-t\right)^{4 \alpha-2} A\left(\frac{x}{\left(t_{0}-t\right)^{\alpha}}\right),
$$

where $t_{0}$ is the time of breakup. The similarity profile $A$ is asymmetric, since there must be a flow across the minimum to produce the Bernoulli suction necessary to sustain the process. The scaling exponent which sets the size of the pinch region is found to be $\alpha \approx 0.687$, just slightly larger than the value of $2 / 3 \approx 0.666$ expected from dimensional analysis, based on the interplay between surface tension and inertia (Eggers \& Villermaux 2008).

The biggest remaining scientific challenge of the field is to understand (and possibly control!) the extremely broad distribution of drop sizes that mark the final stage of the decay into drops (Villermaux 2007). As we have learned to appreciate, this is the result of a series of transformations of the geometry, which require both linear and nonlinear processes to be understood. A number of bright ideas exist, but the dynamics of complex free-surface flows has provided us with an array of wonderful problems for years to come.

\section{References}

Burton, J. C. \& TABoreK, P. 2007 2D inviscid pinch-off: an example of self-similarity of the second kind. Phys. Fluids 19, 102109.

Drazin, P. G. \& ReID, W. H. 1981 Hydrodynamic Stability. Cambridge University Press.

Eggers, J. \& Villermaux, E. 2008 Physics of liquid jets. Rep. Prog. Phys. 71, 036601.

SöDERBERG, L. D. \& Alfredsson, P. H. 1998 Experimental and theoretical stability investigations of plane liquid jets. Eur. J. Mech. B 7, 689-737.

SQuire, H. B. 1953 Investigation of the stability of a moving liquid film. Br. J. Appl. Phys. 4, $167-169$

TAYLOR, G. I. 1959 The dynamics of thin sheets of fluid. Part III. Disintegration of fluid sheets. Proc. R. Soc. Lond. 253, 313-321.

Tammisola, O., Sasaki, A., Lundell, F., Matsubara, M. \& Söderberg, D. 2011 Stabilizing effect of surrounding gas flow on a plane liquid sheet. J. Fluid Mech. 672, 5-32.

Villermaux, E. 2007 Fragmentation. Annu. Rev. Fluid Mech. 39, 419-446.

Villermaux, E. \& Clanet, C. 2002 Life of a flapping liquid sheet. J. Fluid Mech. 462, 341-363. 